Cahiers québécois de démographie

\title{
Immigration et espace urbain
}

Les regroupements de population haitienne dans la région

métropolitaine de Montréal

Immigration and Urban Space

The Formation of Groups of Haitians in the Metropolitan

Region of Montreal

Inmigración y espacio urbano.

Los agrupamientos de población haitiana en el área
metropolitana de Montreal

\section{Francine Bernèche}

Volume 12, numéro 2, octobre 1983

Les migrations

URI : https://id.erudit.org/iderudit/600512ar

DOI : https://doi.org/10.7202/600512ar

Aller au sommaire du numéro

\section{Éditeur(s)}

Association des démographes du Québec

ISSN

0380-1721 (imprimé)

1705-1495 (numérique)

Découvrir la revue

Citer cet article

Bernèche, F. (1983). Immigration et espace urbain : les regroupements de population haïtienne dans la région métropolitaine de Montréal. Cahiers québécois de démographie, 12(2), 295-324. https://doi.org/10.7202/600512ar

\section{Résumé de l'article}

La population haïtienne compte parmi les groupes immigrés les plus récents de la région métropolitaine de Montréal. Dix fois plus nombreuse en 1981 qu'en 1971, cette population d'environ 30000 personnes réside en majorité (80\%) dans trois des quinze municipalités de la région montréalaise : Montréal-Nord, St-Léonard et Montréal. Afin d'identifier les causes et les mécanismes des regroupements de population haïtienne à l'intérieur de ces municipalités, une enquête a été menée au cours de l'été 1982 auprès de 152 ménages haïtiens résidant dans des zones de concentration. Les besoins de ces ménages en matière de logement diffèrent de ceux de l'ensemble des ménages vivant dans les mêmes zones : les ménages haïtiens se caractérisent par un nombre plus élevé de membres et par l'importance accrue des familles comptant des enfants à la maison, enfants qui sont aussi plus jeunes. Les logements qu'ils occupent se situent pour la plupart dans des immeubles à appartements, le plus souvent au sous-sol ou au second étage des bâtiments résidentiels; ce sont surtout des logements de taille moyenne (quatre ou cinq pièces), ce qui donne une densité d'occupation plus forte pour les ménages haïtiens que pour l'ensemble des ménages. La situation socio-économique de ces ménages leur impose également des contraintes particulières, puisque leurs membres actifs sont davantage concentrés dans un même secteur du marché du travail que ne l'est la population active des mêmes zones : les deux tiers sont ouvriers et ouvrières en usine. Ces caractéristiques situent donc la population haïtienne des zones d'enquête parmi les groupes sociaux désavantagés sur le marché du logement et les plus susceptibles d'être touchés par la crise actuelle.
Tous droits réservés @ Association des démographes du Québec, 1983

Ce document est protégé par la loi sur le droit d'auteur. L’utilisation des services d'Érudit (y compris la reproduction) est assujettie à sa politique d'utilisation que vous pouvez consulter en ligne.

https://apropos.erudit.org/fr/usagers/politique-dutilisation/ 
Cahiers québécois de demographie

Vol. 12, no 2, octobre 1983

\title{
Immigration et espace urbain. Les regroupements de population haîtienne dans la région métropolitaine de Montréal
}

\author{
Francine BERNĖCHE*
}

Le Québec est l'une des nombreuses destinations vers lesquelles s'est orientée l'immigration haitienne depuis le debut du $x \times e$ siecle. Tant dans la Caraibe (Republique Dominicaine, Cuba, Bahamas, Martinique, Guadeloupe et Guyane) qu'a l'extérieur de celle-ci (Etats-Unis, Canada, France), les immigrantes et les immigrants venus d'Haiti se comptent aujourdhui par dizaines de milliers.

L'ancienneté de ces différents courants migratoires varie selon leur direction (Hurbon, 1982: 34-35). Les plus anciens datent de la periode de l'occupation americaine en Haiti (1915-1934), fournissant des coupeurs de canne aux plantations américaines de la République Dominicaine et de Cuba. L'immigration haitienne vers le Canada et les Etats-Unis est beaucoup plus récente, débutant sous le régime Duvalier et s'installant véritablement au cours des annés soixante et soixante-dix. Depuis la fin de la derniere décennie, le rythme de cette immigration a nettement ralenti, suite aux politiques restrictives des gouvernements canadien et americain en matiere d'immigration.

Au Quebec, les flux migratoires venant d'Haiti sont longtemps restés plus faibles que ceux issus de la Caraibe anglophone'. Au début des annés 70

* Centre de recherches caraibes, Université de Montréal.

* Cet article se situe dans le cadre d'un projet de recherche dont le responsable administratif est Bernard Bernier et qui a éte subventionné par le Fonds FCAC et le Conseil de recherches en sciences humaines du Canada; des subventions complémentaires ont éte accordés par le Secrétariat d'Etat (Multiculturalisme) et par l'Université de Montréal (CAFIR). Les premières parties du texte comportent des extraits d'un précedent article (Berneche et Martin, 1984). Je tiens a remercier Jean-Claude Martin et Joel Gregory pour leurs commentaires sur une version anterieure du présent article.

1 Cet aperçu de l'évolution de l'immigration haitienne au Québec est tiré des textes suivants: Berneche (1983), Kempeneers et Piché (1983), Normandeau et Piché (1983). 
cependant, le nombre des admissions ${ }^{2}$ en provenance d'Haiti s'est accru de façon marquée, passant de 908 en 1970 a 4856 en 1974 pour finalement supplanter I'immigration de la Caraibe anglophone. Durant la periode 1974-1978, Haiti a ainsi occupé le premier rang des pays sources d'immigration pour le Québec. En 1974,1 'immigration haitienne représentait $14,5 \%$ de l'ensemble de l'immigration au Quebec, mais cette importance relative a par la suite diminué tout comme le volume des entrés venant d'Haiti. En 1980, les 1594 admissions dorigine haitienne ne constituaient plus que $7,1 \%$ de 1 'immigration totale au Quebec.

On caractérise généralement l'immigration haitienne au Quebec en distinguant deux vagues migratoires. La premiere, composé surtout d'immigrantes et d'immigrants venus comme indépendants, date des annés soixante; elle a amené au Quebec des individus le plus souvent qualifiés, professionnels de la santé et de l'enseignement. La seconde vague, celle des années soixante-dix, est tres differente, tant par les catégories dadmission que par les statuts socio-professionnels des individus reçus comme immigrants. A partir de 1975, la plupart d'entre eux entrent au pays comme immigrants désignés ou parrainés. La proportion de femmes dans les flux de ces dernieres annés est devenue de plus en plus forte, accentuant ainsi la surféminité déja manifeste de l'immigration haitienne. La majorité de ces femmes sont ouvrieres dans le textile, tandis que les hommes travaillent surtout dans l'industrie légere.

C'est principalement dans la région métropolitaine de Montréal que 5 'est fixée la population haitienne venant au Québec. Environ $95 \%$ de cette population y résident actuellement, ce qui represente un effectif approximatif de 30000 personnes ${ }^{4}$. Bien qu'ils se distribuent dans tout l'espace montrealais, tant a l'interieur du noyau urbain qu'en perriphérie, les

2 Donnés selon le pays de naissance, ministère des Communautés culturelles et de l'Immigration du Quebec. Ces donnees concernent les personnes ayant un statut d'immigration (indépendant, parrainé ou désigné), enregistrées selon 1 'année d'obtention de ce statut, si celle-ci est postérieure a leur année d'arrivée.

3 Les independants sont sélectionnés selon un ensemble de facteurs, notamment leur formation, leur compétence professionnelle et la présence de parents au Canada. Les parraines sont les proches parents dont un citoyen canadien ou un immigrant reçu prend la responsabilité les designes peuvent etre des parents plus eloignes, mais satisfaisant aux facteurs a long terme applicables aux independants (Kempeneers et Fiché, 1983: 66-67).

4 Selon le recensement de 1981, le Québec compte 25780 personnes nées en Haiti, auxquelles 5 ajoute un effectif d'environ 3500 personnes d'origine haitienne nés au Canada. Seulement $4 \%$ de la population immigrante haitienne au Canada vit a l extérieur du Quebec.

L'importance numérique de la population d'origine haitienne est toutefois sous-estimée par le recensement, comme en témoigne le fait qu'environ $40 \%$ des personnes nées en Haiti se sont déclarées en 1981 d'une origine autre qu'haitienne, française surtout. 
Imigrantes et les immigrants haitiens se retrouvent davantage dans certains secteurs de la métropole, ou l on peut identifier des regroupements de population haitiennes. D'origine relativement récente, une dizaine dannés environ, ces regroupements ne présentent pas encore toutes les caractéristiques qui, dans l espace urbain, permettent de distinguer comme "quartiers ethniques" les concentrations spatiales d'autres groupes d'immigration plus ancienneb.

Dans le cadre d une recherche menée depuis juin 1981, nous avons essayé de degager les causes et les mecanismes entrainant la formation de regroupements de population haitienne dans la région metropolitaine de Montréal. Tout en cherchant a comprendre ce qui amenait des immigrants et des immigrantes a se concentrer dans certaines zones, nous voulions egalement étudier plus a fond la question du logement, en particulier les problemes rencontrés par la population haitienne dans ce domaine. Nous avons ainsi effectue, au cours de 1 'eté 1982 , une enquete qui nous a permis de recueillir une information de base portant principalement sur les conditions de logement et demploi des menages haitiens residant dans les zones de concentration observées.

Aprés quelques reflexions préliminaires sur le theme de l'immigration et la ville, cet article fournit un apercu de la distribution spatiale de la population haitienne dans la région métropolitaine de Montréal, suivi d'un profil general des zones de concentration retenues pour l enquete. Celle-ci est brievement decrite, de meme que certaines caracteristiques des personnes interviewees permettant de les situer dans l'evolution de l'immigration haitienne a Montréal. C'est a partir de résultats issus de cette enquete et de donnés tirés du recensement de 1981 que nous nous proposons, dans cet article, de comparer la situation de la population haitienne dans les zones observees à celle de l'ensemble de la population résidant également dans ces zones. La comparaison porte sur trois aspects principaux touchant a la famille, au logement et a l'emploi. En abordant la question des rapports entre l'immigration et l'espace urbain, nous montrerons l'interet de cette comparaison ainsi que la pertinence des aspects traites.

- Voir à ce sujet le mémoire de maitrise d'André Bastien (Démographie): La Distribution spatiale des Haitiens a Hontréal. Ce mémoire a eté déposé en decembre 1984.

- Far exemple, les quartiers portugais, grec, italien. Les quartiers ethniques ne peuvent etre reconnus comme tels que si existent, dans des espaces où se concentrent des individus ethniquement catégorisés, "des institutions a caractere ethnique, révelatrices d'une organisation communautaire plus ou moins poussé" (Lavigne, 1981: 175). Les institutions issues de la communauté haitienne se localisent pour la plupart dans l'est de l'île de Montréal, a l'intérieur dun périmetre assez. vaste; par ailleurs, il s'en crée de plus en plus a Montréal-Nord, dans la partie est de la ville où se retrouvent les plus fortes concentrations de population haitienne. 


\section{IMMIGRATION ET ESPACE URBAIN}

L'immigration dans les pays industrialisés est principalement un phénomene urbain ${ }^{7}$, cette caractéristique étant encore accentué par la crise économique des dernieres annees (Alalut, 1982: 5). L'installation dans une ville etrangere fait partie du processus migratoire de beaucoup d'hommes et de femmes qui, venant de formations sociales differentes, se retrouvent dans des espaces urbains ayant deja leur propre histoires. Ainsi, dans le cadre général de l analyse de ia reproduction de la force de travail, "les travailleurs immigres sont le plus souvent perçus a travers la problematique de leur insertion dans un tissu urbain dont la forme est plus ou moins preexistante" (Foche, 1975: 15j. Les strategies de survie adoptés par les migrants et les migrantes dans les centres urbains apparaissent aussi comme l un des aspects significatifs de la migration (Fortés et Walton, 1981: 59).

La recherche d un logement est, avec celle d un emploi, l une des premieres préccupations des immigrants et des immigrantes qui arrivent dans une ville étrangere. De prime abord, le logement constitue l un des principaux enjeux dans 1 insertion urbaine des groupes immigres, insertion qui se fait dans un espace socialement differencie, marque par la discrimination ethnique et raciale. "La question du logement devient ainsi un élément important de la dynamique des rapports sociaux entre societé d'accueil et groupes immigres" (Borgogno, 1978: 43 ).

Les rapports sociaux qui s'expriment dans la ville modelent et transforment la répartition residentielle des individus et des groupes d'individus (Castells, 1977: 231). La population immigrante participe a ces rapports a la fois comme force de travail et comme consommatrice de ce produit particulier qu'est le logement. La place que cette population occupe dans les rapports de production et dans la distribution du produit-logement détermine largement sa répartition dans lespace urbain; mais ce sont les rapports complexes lies entre autres a l'ethnicité, a la langue et à la couleur de la peau qui permettent d'en préciser les modalites. Comme la population immigrante n'est pas homogène, son insertion dans le milieu urbain se différencie suivant les pays dorigine et suivant les couches sociales de chaque pays. Il y aurait cependant davantage de segrégation entre les couches aisés et les couches populaires qu entre les membres de groupes ethniques differents partageant les memes difficultes economiques (Lee, 1977: 26).

7 Le milieu rural est aussi concerne par l immigration de travailleurs et de travailleuses agricoles ne disposant souvent que de permis temporaires, mais c'est en milieu urbain que se concentre la majorité des immigrants et des immigrantes.

- L'histoire specifique de Montreal a déja imprimé dans la trame urbaine une division issue du rapport de force entre anglophones et francophones, les premiers résidant dans l'ouest et les seconds, dans l'est de l'ile de Montréal.

- Le theme de l'articulation des rapports sociaux en milieu urbain est traité de façon plus détaillée dans un autre article: Berneche et Martin, 1984: 6-9. 
Si la trajectoire urbaine de plusieurs groupes immigrés ou de fractions importantes de ces groupes a "tendance a suivre celle des couches modestes de la societé", elle presente par ailleurs des caracteristiques qui lui sont propres, cumulant iau niveau de l'habitat comme au niveau de l emploi, le maximum d'inconvenients présentés par cette trajectoire" (Barou, 1980: 17). Les groupes immigres connaitraient donc une double situation:

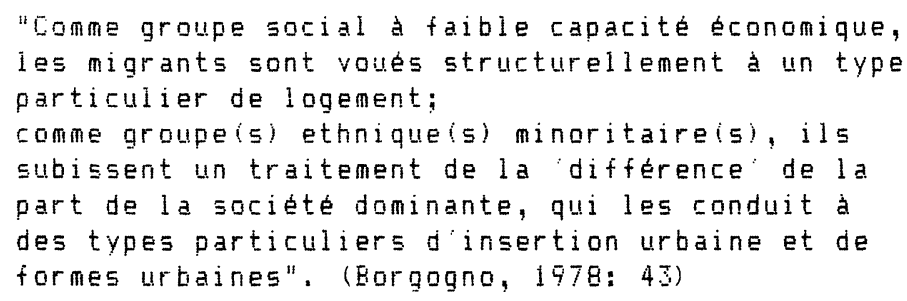

Áinsi, les "mécanismes 'naturels' de la penurie et de la ségrégation économique" régissant le marché du logement meneraient progressivement au "refoulement vers des territoires specifiques de groupes 'specifiques'" (Eorgogno, 1978: 52 ).

La formation de "quartiers ethniques" donne par ailleurs lieu d de multiples interprétations. Vue par plusieurs auteurs comme une "question d'appartenance ethnique et de culture" (Folese, Hamel et Bailly, 1978: 28), elle est considérée par d'autres plutót comme une création de la bourgeoisie ou de la petite bourgeoisie ethnique qui y trouve une base daccumulationio (Bernier, 1978: 210-211) et les assises de son pouvoir (Lavigne, 1981: 178-181), ou encore comme une réaction de la population immigré face aux situations que lui impose la stratégie urbaine des classes dominantes (Barou, 1980: 3-4). Complexe, ia problematique de l'insertion urbaine des groupes immigres touche donc des questions fort importantes qui concernent a 1 a fois la gestion de l'immigration et les enjeux de l'espace urbain.

Ces quelques réflexions fondent les questions précises abordés dans le cadre de cet article. Les regroupements de population haitienne dans la région montréalaise se trouvent-ils dans des zones marqués par des traits communs, qui leur sont spécifiques? Les ménages haitiens qui résident dans ces zones occupent-ils une place particulière dans l ensemble de l'immigration haitienne au Quebec? La localisation résidentielle de ces menages se rapproche-t-elle de celle de la population montrélaise ayant le meme profil socio-économique ou traduit-elle surtout la situation d'une population immigrante qui, par le biais de la discrimination et du recours aux réseaux, en vient a se regrouper dans certaines zones?

Le premier theme traite donc diune double question: celle de la spécificité des territaires ou se retrouvent les groupes immigres et celle de

10 "Mais un quartier ethnique peut aussi etre crée sous la pression dune bourgeoisie non ethnique qui, en utilisant la "visibilite" dun certain groupe, crée un quartier ethnique" (Bernier, 1978: 211 ). 
l'influence du cadre bati sur leur type d'insertion. On ne peut interpréter le mode de consommation résidentielle de la population immigrante sans tenir compte du stock de logements offerts dans les zones où elle se concentre; c'est ainsi en effet que peut etre évalué la position relative des ménages immigrants parmi les clienteles du marché du logement et que peuvent etre aussi etablis des liens entre leur distribution spatiale et certains types de bati résidentiel (Lee, 1977: 35 ).

Le second theme concerne les caractéristiques menes de la population haitienne résidant dans les zones denquête. Le bref aperçu qui a été donné de l'évolution de l'immigration haitienne au Québec a deja souligné les changements qui, au cours des annés, ont modifié la composition de cette population immigrante. Il importe donc de voir si les ménages haitiens qui se concentrent dans certaines zones de la région montréalaise se situent également de façon particuliere dans l'ensemble de l'immigration haitienne, tant en ce qui concerne leur période d'arrivé que leur statut d'entré et leur structure professionnelle.

Enfin, c'est la question de la place occupée dans la structure sociale par la population haitienne "regroupée" qui est abordée en comparant sa situation a celle de l'ensemble de la population résidant dans les memes zones. Le choix des caractéristiques observés tient compte de trois dimensions: les besoins en matière de logement, les conditions d'habitation et les ressources économiques. Elles permettent de situer la population haitienne par rapport a une population qui partage le meme espace, en identifiant dans quelle mesure se trouve exprimée sa position particulière de population imigrante.

Les premieres caracteristiques observées, soit la structure par age et la structure failiale des populations concernés, traduisent linfluence du "cycle de vie" des ménages sur le choix d'un logement (Polese, Hamel et Bailly, 1978: 12); elles fournissent des indices sur leurs besoins en matiere d'habitation et sur leur position relative sur le marché du logement. Ainsi, 1a longue 1 iste des groupes sociaux pour qui l'obtention d'un logement locatif devient de plus en plus difficile (rareté des logements a bon marché, discrimination) inclut, outre les travailleurs immigrants et les assistes sociaux, les mères célibataires et les familles avec enfants (choko, 1980: $270-2721$.

Une autre caractéristique démographique, la taille des aenages, sert a évaluer les conditions d'habitation de ces ménages, sur la base de leur densité d'occupation diun logement (nombre de personnes/nombre de pieces) 11 . Le mode d'occupation d'un logement (location ou propriete), le type de batiment résidentiel, l etage habité ainsi que le nombre de pieces constituent autant de variables qui permettent de caractériser la consommation residentielle des ménages. Les types de logeant occupes par ces menages comportent en effet des avantages et des inconvenients qui sont lies a la satisfaction de leurs besoins

11 Cet indicateur de la situation du logement présente cependant plusieurs limites, car il ne tient pas compte de la dimension ou de la division des piéces, ni de l'age ou du sexe des personnes y résidant (Choko, 1981: 114 ). 
et qui soulignent le caractere plus ou moins favorable de leur position sur le marché du logement. Les possibilités d'acces a ce marché et a celui de l'emploi comptent dialleurs parmi les principaux determinants de la concentration résidentielle (Lee, 1977: 27).

Les regroupements spatiaux s'operent "en fonction de la capacité sociale des sujets, c'est-a-dire, dans le systeme capitaliste, en fonction de leurs revenus, de leur statut professionnel, du niveau dinstruction, de l'appartenance ethnique, de la phase du cycle de vie, etc." (Castells, 1977: 218). Les variables économiques sont donc étroitement liées à la ségrégation urbaine, qui est d'abord sociale (Barou, 1980: 17). Le choix d'un logement est ainsi soumis a la contrainte du revenu, la hausse des loyers constituant - dans les cas de restauration notamment - l'instrument sélectif principal de la population occupante" (Barou, 1980: 11). Deux caractéristiques, soit le nombre de enores actifs dans les familles et les types d'emploi parmi la population active, fournissent une appréciation indirecte des possibilités financieres des ménages. Elles permettent de voir comment la ségrégation économique rassemble dans une aire commune des individus ayant un profil semblable, tout en montrant que léventail des choix résidentiels est, pour plusieurs immigrants et immigrantes, fort restreint. Le fait detre cantonnés dans certains types d'emploi les force en effet a trouver des logements a prix modestes, tout comme leur structure démographique les contraint a rechercher des types particuliers de logement (Lee, 1977: 27).

Dans le cadre de cet article et en raison de la disponibilité des donnés statistiques, l'analyse des themes proposés se trouve évidemment limitée et portera surtout sur une situation d'ensemble, a l'échelle des zones d'enquete.

\section{DISTRIBUTION SPATIALE DE LA POPULATION HAÏTIENNE}

Afin de mieux situer les zones de concentration retenues pour l enquete, nous donnons en premier lieu un bref aperçu de la distribution spatiale de la population hajtienne dans la région métropolitaine de Montréal. Selon le recensement, 3095 personnes originaires d'Haiti résidaient dans cette région en 1971. Cette population était alors légerement concentrée dans l'espace métropolitain et se trouvait moins distante de la communauté francophone que de la communauté anglophonei2. En 1981, cette population est presque dix fois

12 Le coefficient de ségrégation de la population née en Haiti par rapport a la population totale de Montréal (R.M.R.) en 1971 est de 0,61 ; il est de 0,63 par rapport aux francophones et de 0,77 par rapport aux anglophones. PLus le groupe observé est concentré, plus ce coefficient tend vers 1 (Folese, Hamel et Bailly, 1978: 19-23). 
plus nombreuse (24 295 personnes nées en Haiti) et reste encore trés légerement concentrée par rapport a l'ensemble de la population metropolitaine (coefficient de ségrégation: 0,58$)^{13}$.

Selon le recensement canadien de 1981 , pres de $80 \%$ des personnes nees en Haiti resident dans trois des quinze municipalités de la région montréalaise. Ainsi la ville de Montréal ou se retrouve le tiers de la population metropolitaine compte par ailleurs la moitié $(52 \%)$ de la population provenant d'Haiti. Cette population se concentre davantage dans les municipalites de Montréal-Nord et de St-Léonard qui reçoivent le quart $(27 \%)$ de ses effectifs, une proportion quatre fois superieure a celle représentée par la population de ces deux villes dans l'ensemble montréalais.

Les residentes et résidents haitiens ne se distribuent pas également sur le territaire de chacune de ces municipalités. A Montréal, c'est principalement dans les quartiers $5 t-M i c h e l$ et Farc Extension, et secondairement dans ceux de Villeray, Rosemont, Côte-des-Neiges et Rivière-des-Frairies que se localise surtout la population née en Haiti, selon le recensement de 1981. La partie est de montreal-Nord rassemble la majorité des menages haitiens de cette municipalité, alors que ceux résidant a st-Léonard se partagent largenent en deux zones, situees au nord du boulevard Métropolitain et bordant les limites est et ouest de la ville.

Cet aperçu de la distribution spatiale de la population haitienne dara region montrealaise provient de donnees qui n etaient pas disponibles au monent de notre enquete. C'est donc a partir de sources secondaires ${ }^{4}$ qu ont été choisies et délimitees les zones d'enquete. Ces zones correspondent, dans leur ensemble, aux principaux regroupements identifies par le recensement de 1981.

\section{CARACTÉRISTIQUES DES ZONES D'ENQUÊTE}

Trois grandes zones ont été retenues pour l enquete; ce sont ceiles de Montreal (districts St-Hubert et St-Michel), St-Léonard et Montreal-Nord (Lapte 1). Ces zones ont eté choisies en fonction des indices de concentration

13 Le calcul a été fait a partir de compilations spéciales, fournies au 0. de montreal de 3 tatistique Canada en janvier 1984. La jistribar: observee est celle de la population nee en Haiti par secteur de recensation de la k.M.R. de Montréal. Meme si elle néglige les personnes d oriçne naitienne nees au Canada, cette distribution est préferable a celle établie selon 1 origine ethnique qui sous-estime fortement 1 'ensemble de catte population. Notons que l'utilisation du lieu de nassance pour identifier la population haitienne exclut une proportion beaucoup plus importance de personnes d'origine haitienne en 1981 qu'en 1971 , ce qui rédust la portee du coefficient calculé pour 1981.

14 Recensements municipaux de Montréal-Nord (1981), de 5t-Léonard (1978) et de Montréal (1978); données de la CECM (1978-1982); recensement du Canada (1971). 


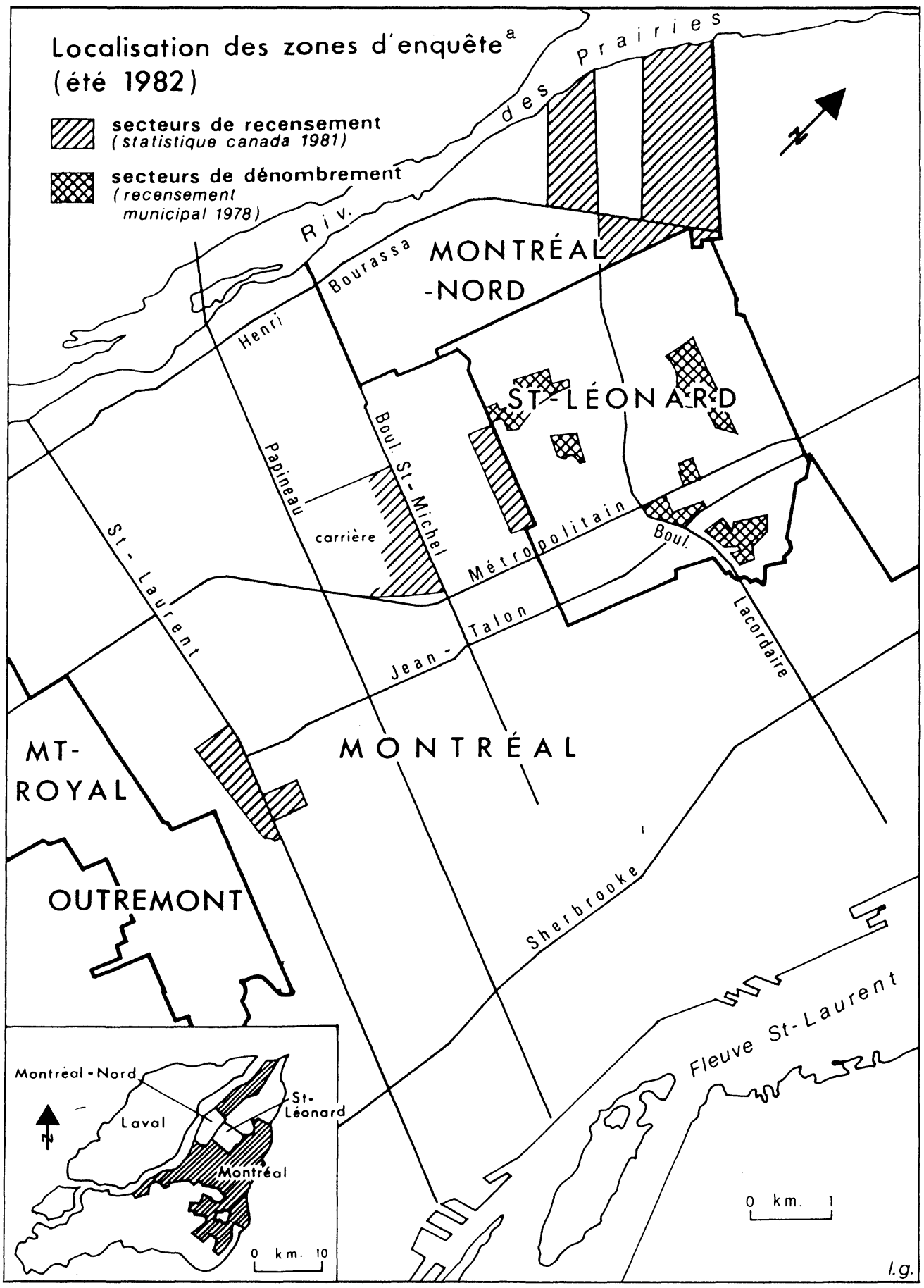

a Cette carte est tirée d:un article précédent (Rernèche et liartin, 1984: 12). 
obtenus dans chacune des municipalites considerees ${ }^{15}$. Afin de voir si elles occupent une position particuliere dans l'espace montréalais, nous caracteriserons les zones d'enquete en soulignant certains aspects de leur cadre bati résidentiel (composition, ancienneté, qualité) et en tenant compte de la population immigrante qui habite sur leur territoire. La description des formes de l'espace residentiel permettra également de mieux cerner les traits spécifiques de la consommation résidentielle des ménages haitiens concentres dans ces zones.

Le cadre bati résidentiel se differencie nettement diune zone a 1 'autre. Ainsi, les batiments residentiels de type "plex" (duplex, triplex, multiplex)

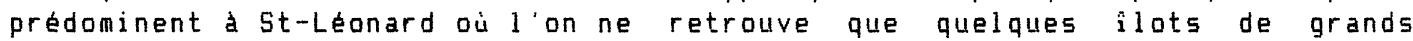
ensembles immobiliers $(12$ appartements et plus). La situation s'inverse dans la partie est de Montréal-Nord qui comporte principalement des immeubles a appartements, de taille variable. Dans la zone de Montréal, le cadre bati est beaucoup plus dense et hetérogene; il est composé surtout dimmeubles en rangé, a la différence des deux autres zones où les immeubles sont souvent jumelés ou détachés.

Le parc de logements est manifestement plus ancien dans la zone de Montréal, ou les trois-quarts des batiments résidentiels inclus dans le district St-Hubert (á proximité de Farc Extension) ont eté construits avant 1946 et la moitié de ceux de St-Michel lont eté entre 1946 et 196016.

15 Farmi les indices possibles, nous avons retenu un indice simple déa utilisé par T.R. Lee (1977: 171-172), soit:

$$
\text { L.Q.1 }=\frac{s_{1}}{5}, \frac{t_{1}}{\mathrm{~s}}
$$

oú L.Q.1 est le quotient de localisation pour la zone i;

5. est la population du groupe ethnique considerédans la zone i;

$S$ est la population totale du groupe ethnique consideré;

$t_{1}$ est la population totale de la zone i;

$T$ est la population totale.

Une zone ayant un indice supérieur a 1 peut alors etre considérée comme une zone de concentration pour le groupe ethnique etudié. Les zones d'enquete se composent de secteurs de recensement (SR) ayant des indices superieurs a 1,3 pour Montréal-Nord ( $5 S R$ ) et a 2,3 pour Montréal ( 4 SR retenus parmi les 11 SR initialement prévus). A $5 t$-Léonard, 23 secteurs de dénombrement, plus petits que les $5 R$, ont eté choisis a partir des indices $(5 e u i 1: 1,6)$ et d'autres criteres (contiguité). Des raisons d'ordre pratique, lieses au modele d'échantillonnage ainsi qu'a la disponibilité des donnés, ont motivé la réduction de la zone d'enquete a Montréal ainsi que le choix de secteurs plus restreints à St-Léonard.

16 Données du recensement de 1981. Toutes les données sont tirés de deux bulletins portant sur les caractéristiques de la population dans la région métropolitaine de Montréal par secteur de recensement (cat. $95-918$ et 95-959) et des bandes sommaires de statistique Canada (secteurs de denombrement). 
Dans les zones de Montréal-Nord et de St-Léonard par contre, presque tous les immeubles résidentiels ont été batis apres 1960 , surtout durant la période 1971-1981. Un peu plus de la moitié (53\%) des logements situés dans l'est de Montréal-Nord et $67 \%$ de ceux localisés dans la zone d'enquéte de St-Léonard datent en effet de cette période.

La moitié des batiments résidentiels évalués a Montréal-Nord et a st-Léonard peuvent etre considérés en très bon état ${ }^{7}$, comparativement au cinquieme seulement de ceux situés a Montréal. Selon le recensement de 1981 , environ $15 \%$ des logements situes dans les deux premieres zones nécessitent des reparations (mineures ou majeures), alors que cette proportion s'éleve a $33 \%$ dans les secteurs retenus a Montréal. Les facteurs d'usure associés a l'age des batiments jouent sans doute davantage dans cette derniere zone ou l'ancienneté du bati et certains facteurs environnementaux peuvent avoir accéléré le processus de détérioration résidentielle.

Il ne semble donc pas y avoir de traits communs differenciant nettement les trois zones d'enquête dans l'espace bati de la région montréalaise. Ce sont plutôt les caractéristiques propres a chacune qui transparaissent, suivant l'insertion de ces zones dans la chronologie du développement résidentiel. Dans l'ensemble toutefois, leur profil résidentiel se rapproche davantage de celui de la ville centrale que des zones périphériques de l'île ou des banlieues nord et sud. En témoigne la faible importance de l unifamiliale isolée qui ne dépasse pas $6 \%$ du stock de logements dans chaque zone d'enquête, ce qui est très proche du $5 \%$ observé dans l'ensemble de la ville de Montréal, mais loin des $27 \%$ de logements "unifamiliaux isolés" a l'échelle métropolitaine en 1981 .

Diverses quant a leur espace bati, les zones d'enquete partagent une premiere caractéristique sur le plan démographique: 1 importance de la population immigrante. En effet, la proportion des personnes nées hors du Canada est, dans chacune des zones, supérieure a $16 \%$, proportion représentée dans l'ensemble de la région metropolitaine. Cette proportion varie cependant d'une zone a l'autre, de meme que la part des immigrantes et immigrants haitiens dans l'ensemble. Dans la zone de $5 t$-Léonard, prés du tiers de la population vient de l'exterieur du Canada, comparativement au quart dans la zone de Montréal et a un peu moins du cinquième dans celle de Montréal-Nord.

La population immigrante des zones d'enquete se compose d'un fort pourcentage de personnes originaires de pays européens, d'Italie surtout, soit $70 \%$ a St-Léonard, $60 \%$ a Montréal et $48 \%$ a Montréal-Nord. Les personnes nées en

17 Les batiments où résidaient les personnes interviewés ont fait l'objet d'une évaluation externe, effectuée dans le cadre du projet. Cette évaluation porte toutefois davantage sur l'apparence des batiments que sur leur structure meme. Nous avons retenu les composantes suivantes: fondation, murs, portes et fenetres, balcons et galeries, avant-toit. 
Haiti représentent environ le cinquieme de cette population dans les deux premieres zones, mais constituent $41 \%$ des effectifs immigrants dans la troisieme. L'importance relative des immigrantes et immigrants haitiens parmi la population totale demeure toutefois restreinte, variant selon les zones de $5 \%$ (Montréal) a $6 \%$ (5t-Léonard) et $7 \%$ (Montréal-Nord); même faibles, ces proportions dépassent largement celle de $0,9 \%$, valable pour l'ensemble de la région metropolitaine.

Selon les donnés du recensement, $18 \%$ de la population immigrante haitienne residaient dans les zones d'enquete en 1981, alors qu'on y retrouvait seulement $2 \%$ de la population métropolitaine. Les résultats de l enquete nous fournissent des indices sur la place occupee par cette population "regroupee" dans l'ensemble de l'immigration haitienne. Avant de présenter ces résultats, nous decrirons brievement 1 'enquete ainsi que l'information de base qui a été recueillie.

\section{ENQUÊTE ET INFORMATION DE BASE}

Les résultats présentés dans cet article sont tirés d une enquete menee au cours de 1 'été 1982 aupress de 152 ménages haitiens. L'importance relative des entrevues réalisées dans chacune des zones d'enquete correspond a 1 a distribution spatiale de l'ensemble des ménages haitiens parmi ces zones, établie a partir de différentes sources statistiques. Ainsi 62 entrevues ont été complétés dans la zone située a Montréal-Nord $(40,8 \%$ de 1 ensemble), 51 1 ont éte dans celle de St-Léonard $(33,5 \%)$ et 39 dans celle de Montréal $(25,7 \%)$.

Afin dobtenir un ensemble de 150 questionnaires complétés, nous avons tiré un échantillon aléatoire systématique d'un ménage sur quatre parmi les ménages haitiens identifiés dans chacune des zones denqueteia. Le taux de réponse obtenu s'avere trés satisfaisant pour une enquete menée aupres d une populatior composée surtout d'immigrantes et d'immigrants récents; un peu plus de la moitié des personnes contactés $(55,3 \%)$ ont accepté de repondre au questionnaire proposé.

L'unité de base de l'échantillon était le ménage, défini comme "une personne ou un groupe de personnes occupant un meme logement"io. Au sein de chaque menage, la personne a rejoindre etait celle que lion considerait responsable du logement ou l'une des responsables 20 ou encore son conjoint, quels que soient l'age, le sexe ou la situation matrimoniale de la personne répondante.

1 L Le modéle d'échantillonnage a été établi en consultation avec Denise Granger, responsable de recherches au Centre de sondage de l'Université de Montréal.

19 Dictionnaire du recensement de 1981: 75.

20 La personne qui a signé le bail est considerée comme responsable du logement. Si la/le signataire n'habite pas le logement, la personne considerée responsable est celle qui paie le loyer ou, a defaut, fait affaire avec le propriétaire en cas de problèmes. 
Les personnes interviewés devaient répondre a l'un ou l'autre des deux types de questionnaire établis pour l'enquete. Le premier, restreint et précodé, était adressé a trois ménages sur quatre; le second, ouvert et enregistré, rejoignait le quatrième ménage. Le premier questionnaire était en français 21 et durait environ une heure; le second, dune durée approximative de deux heures et demie, se faisait en creole ou en français selon le choix de la personne interviewé. Outre les caracteristiques générales des individus et des ménages, les themes abordés portaient sur leur histoire résidentielle et le logement actuel, leur situation d'emploi (passéet actuelle) ainsi que sur leur venue au Canada. Des observations étaient également demandés sur la zone de résidence et sur la vie a Montrél, en particulier sur les problemes rencontrés. Le second questionnaire mettait davantage l'accent sur les reseaux de parents et d'amis, l'utilisation des commerces et des services ainsi que sur la participation à differents types d'activites (associations, loisirs) de la communauté haitienne.

\section{CARACTÉRISTIQUES DES PERSONNES INTERVIEWÉES}

La population interviewee se compose de 152 personnes au total. Cette population compte une proportion légèrement plus elevé de femmes que d'hommes $(55 \%$ comparativement a $45 \%)$, attribuable au fait que le cinquieme des ménages ont pour responsable un parent seul, ce parent étant presque toujours une femme. Ainsi, bien que la majorité des personnes interviewées soient mariées ou vivent en union consensuelle, il existe une difference notable selon le sexe: ces deux catégories matrimoniales comprennent en effet $83 \%$ des hommes, mais seulement $57 \%$ des femmes. Par ailleurs, $29 \%$ des femmes interviewés sont séparées, divorcées ou veuves comparativement a $7 \%$ des hommes. on observe aussi parmi l'ensemble des membres des ménages (623) une légère surféminité, due surtout a la représentation plus forte des femmes haitiennes ages de 50 ans et plus $(5,3 \%)$ que des hommes du meme age $(1,8 \%)$. Liée à la mortalité différentielie selon le sexe, cette caractéristique peut etre davantage encore associée a la surféminité de l'immigration haitienne et a son accentuation au cours des dernieres annés, phénomene qui se trouve en partie expliqué par "le type d'emplois pour lesquels ces femmes sont recrutees" (Kempeneers et Piché, 1983: 45$)$ et par la venue relativement récente de femmes plus ggés, parentes de familles immigrées a Montréal et parrainés par elles.

Au moment de l'enquete, les personnes interviewées residaient depuis un certain temps déjà au Québec, puisque leur durée moyenne de sejour atteignait presque huit annés. En fait, la moitié des répondants et des repondantes sont arrivés entre 1971 et 1974 , principalement comme touristes (Tableau 1). Un peu plus du quart sont venus au cours des annés 1975 et 1978; il s'agit surtout d'immigrants et d'immigrantes parrainés. Dans l'ensemble, seulement $14 \%$ des personnes interviewés sont entrés au canada avec un statut dimmigrant independant. Les individus qui ont été parrainés sont deux fois plus nombreux $(34 \%)$ et ceux venus comme visiteurs ou étudiants, trois fois plus représentés $(52 \%)$.

21 Si la personne ne pouvait parler que le créle, un interviewer ou une intervieweuse faisait passer le questionnaire en creole. 
TABLEAU 1

Période d'arrivée et statut d'entrée au Canada des immigrantes et immigrants haitiens interviewes dans l'enquete

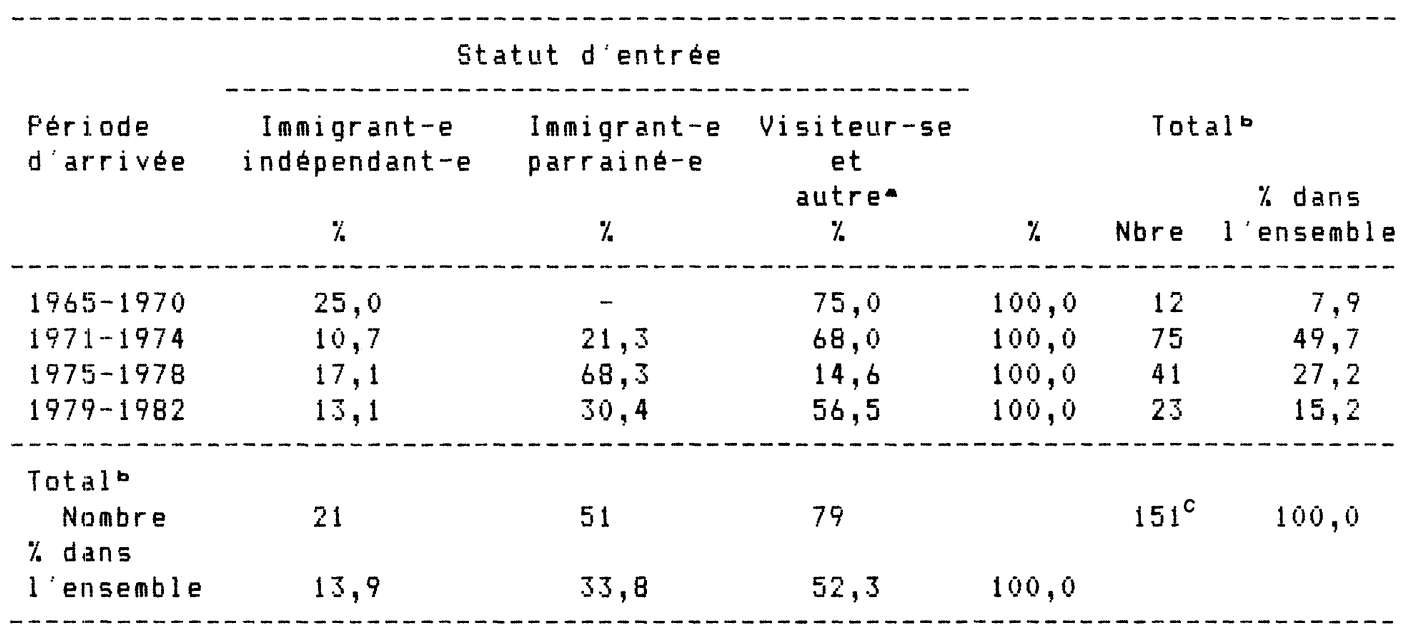

Source: Données d'enquete, été 1982.

- Comprend huit étudiants et étudiantes ainsi qu'un travailleur temporaire.

- La marge d'erreur d'echantillonnage autour d'une proportion de $50 \%$ pour 1 'ensemble de l'échantillon $(N=152)$ est de $\pm 8 \%$.

- Il y a un cas manquant (homme).

Une grande partie des repondants et des répondantes se sont trouvés concernés par les changements introduits dans la loi canadienne d'immigration depuis 1972. Ainsi, au moment de la mise en viqueur des nouveaux reglements, tous les individus entrés comme visiteurs et qui n'étaient pas immigrants reçus ont dû régulariser leur statut. C'etait le but de l'operation Hon Pays menée par le gouvernement fédéral en 1973-1974. En 1980, un programme spécial d'amnistie visant la population haitienne a aussi étémis sur pied et a rejoint depuis, plus de 4000 personnes 22 .

La population considérée dans l'enquete appartient donc en majorite a la seconde vague de l'immigration haitienne au Quebec: celle des annees 70 . La structure professionnelle de la population interviewée montre également qu'elle partage, en termes demplois, les caractéristiques générales des immigrantes et immigrants haitiens arrivés au cours de ces années. En effet, $80 \%$ des répondants et des répondantes occupant ou ayant déja occupé un emploi sont ouvriers et ouvrieres en usine ou travaillent comme employés-es dans les services. En comparant la situation de la population haitienne résidant dans

22 Pour de plus amples renseignements sur la situation particuliere de la population haitienne, voir le livre de F. Dejean (1978). 
les zones d'enquete a celle de l'ensemble de la population vivant dans les memes zones, nous verrons comment cette structure professionnelle traduit l'insertion spécifique sur le marché du travail de la population haitienne "regroupée".

\section{SITUATION COMPARÉE}

Dans les zones denquete, la population haitienne fait partie des groupes immigrés les plus récents, ce qui donne une importance particulière à 1 'aspect immigration dans l'étude de la situation familiale et socio-économique des ménages haitiens. Differente par la culture et la couleur de la peau, cette population s'insere dans un espace urbain marque non seulement par la discrimination sociale, mais aussi ethnique et raciale. C'est également une population créolophone qui se retrouve dans un milieu a majorite francophone, utilisant un autre langage pas toujours bien compris.

Le comportement résidentiel des personnes interviewées dépend donc a la fois de leur histoire spécifique en Haiti et de leurs experiences multiples depuis leur arrivé a Montréal. Dans quelle mesure ce comportement traduit-il leur situation particuliere comme membres d'un groupe immigré ou reflete-t-il leur rapprochement avec l'espace social environnant? C'est ce que nous essaierons de voir en comparant certaines caractéristiques demographiques et socio-économiques de la population considérée dans l'enquete et de la population totale se trouvant dans les limites des zones observes.

\section{Structure par lge et structure de la famille}

Les membres des menages haitiens faisant partie de l'enquete, soit 623 personnes au total, composent une population jeune, beaucoup plus jeune que l ensemble de la population résidente. En effet, les moins de 15 ans representent $39,5 \%$ de ses effectifs et 1 es 65 ans et plus, seulement $2,4 \%$. Ces proportions sont respectivement de $22,7 \%$ et de $5,5 \%$ pour la population totale. $L$ 'importance relative des 25-34 ans et des 35-44 ans, groupes situés au coeur de la population active, est par ailleurs fort semblable. La charge des personnes dépendantes du fait de leur jeune age s'avere donc beaucoup plus lourde pour les familles haitiennes.

Les familles sans enfants a la maisonz3 représentent un tres faible pourcentage $(6,7 \%)$ des 149 familles haitiennes touchees par 1 enquetez4 (Tableau 2), alors que celles comptant un ou plusieurs enfants agés de moins de

23 Suivant les definitions de Statistique Canada en 1981. Une falle de recensenent comprend des personnes ayant entre elles des liens conjugaux ou de filiation; un enage peut etre non familial, unifamilial ou multifamilial. Les enfants sont les fils et filles qui ne se sant jamais mariés, peu importe leur age, et qui vivent dans le meme logement que leurs parents (Dictionnaire du recensement de 1981: 65,70 et 76 ).

24 Pour être comparée aux données tirées du recensement, l'information doit porter sur les familles plutôt que sur les ménages. 
15 ans constituent $82 \%$ de l'ensemble. On retrouve, parmi la population totale, une proportion quatre fois plus importante $(28,0 \%)$ de familles sans enfants a la maison, mais des pourcentages tres peu differents pour les familles avec un ou deux enfants, se situant autour de $25 \%$ a $30 \%$. C'est donc chez les familles plus nombreuses, celles ayant trois enfants ou plus, qu'il faut chercher la difference marquante entre les deux populations: leur importance relative parmi la population haitienne est de $32,9 \%$, le double de celle observée parmi la population totale $(15,6 \%)$ en 1981. Cette caractéristique laisse deviner la pression qui peut s'exercer sur ces familles dans la recherche d'un logement (chambres pour les enfants, espaces de jeu, risques de bruit pour les voisins, etc.).

Les ménages haitiens sont, pour les neuf-dixièmes d'entre eux, des ménages familiaux, tels que définis par statistique Canadazs (Tableau 2). La proportion des ménages non familiaux, qui s'est rapidement accrue dans la région montréalaise au cours des dernières années (Mathews, 1978: 13), est d'ailleurs nettement plus forte parmi la population totale $(22,0 \%)$ que parmi la population haitienne $(10,5 \%)$. Celle-ci se compose surtout de ménages de type "famille nucléaire" (avec ou sans d'autres personnes), qui représentent $59 \%$ de l'ensemble des ménages haitiens.

Une fraction importante des menages haitiens, soit un peu plus du quart $(27,6 \%)$, comptent au moins une famille monoparentale 26 . On dénombre ainsi 48 familles monoparentales dans l'ensemble de l'enquête; pour 33 d'entre elles, il s'agit de la famille de l'une des personnes interviewes en tant que responsables du logement qu'elles occupaient. Contrairement a ce qui a eté observé pour les couples sans enfants a la maison, l'importance relative des familles monoparentales parmi les familles haitiennes $(32,2 \%)$ est plus ellevé que celle estimée pour l'ensemble des familles résidant dans les zones d'enquete $(19,6 \%)$. Cette remarque concerne aussi chacune des zones.

Tant parmi la population haitienne qu'a l'échelle de toute la population, les personnes en charge de familles monoparentales sont presque exclusivement des femmes, puisqu'elles representent le parent unique pour 45 des 48 familles monoparentales de 1 'enquete et pour $88 \%$ de toutes les familles monoparentales résidant dans les méme zones. Ces familles partagent également une autre caracteristique: leur situation économique difficile, comme lindiquera le nombre d'actifs selon la structure familiale.

L'interprétation des ressemblances et des differences observees est complexe et doit, de toute évidence, tenir compte de la situation particuliere de la population haitienne. Cette situation peut en effet etre liéd la

25 Comme la définition de fallie de recenseant exclut les personnes nayant pas de liens conjugaux ou filiaux, un frère et une soeur vivant dans le meme logement ne constituent pas un ménage familial.

26 Four definir la famille monoparentale, nous avons tenu compte de l'absence effective d'un conjoint dans le logement, quel que soit l'état matrimonial du parent seul. 
TABLEAU 2

Caractéristiques des ménages (nombre de familles et de personnes) et des

familles (nombred'enfants a la maison) pour les ménages haitiens de

1 'enquete et pour 1 'ensemble des ménages résidant dans les mémes zones

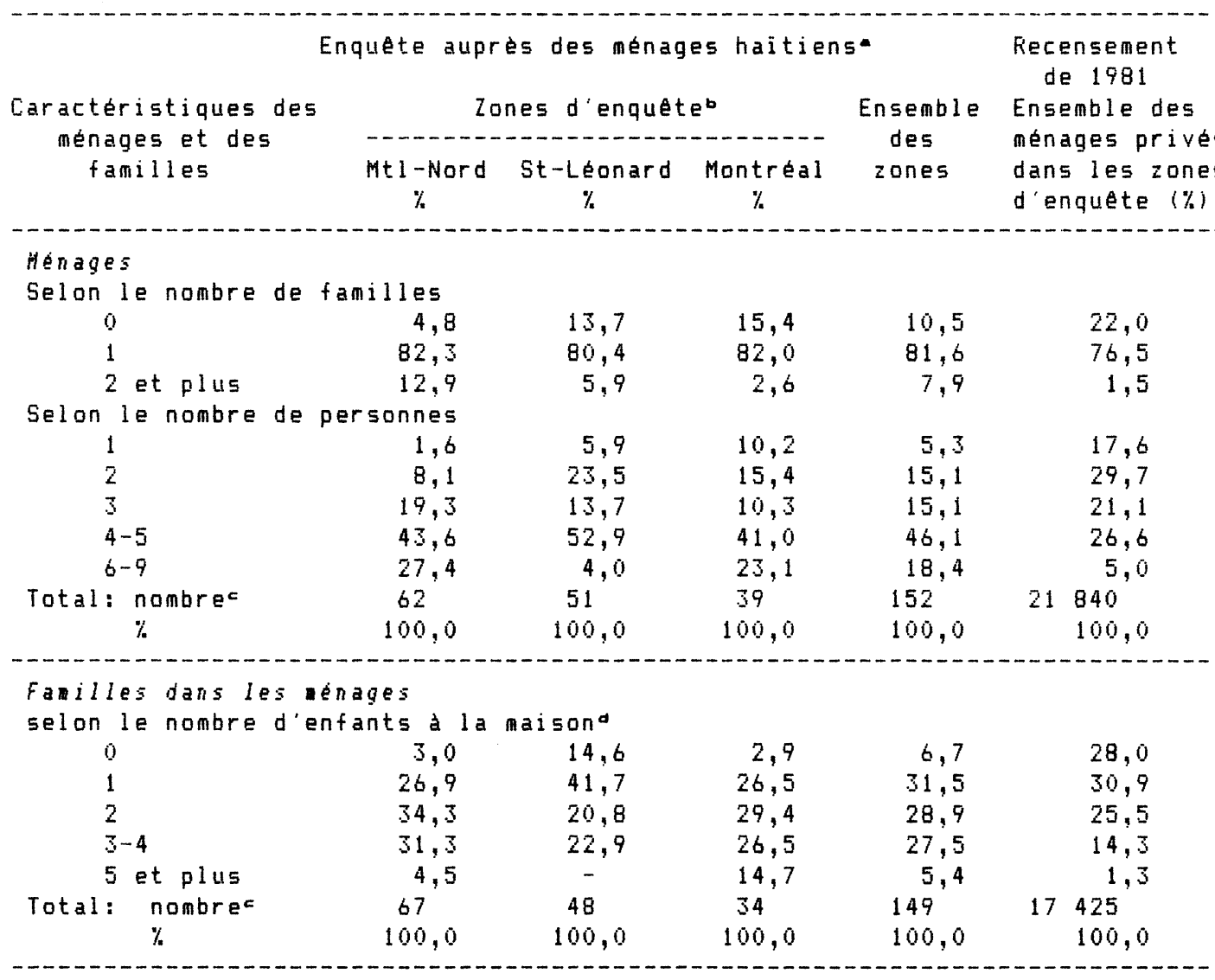

Sources: Donnés d'enquête, été 1982. Statistique Canada, Fecensement du Canada, 1981, catalogues nos $95-918$ et $95-959$ (secteurs de recensement); bandes sommaires (secteurs de dénombrement).

- Ménages dont le ou la responsable est d'origine haitienne, i.e. né-e en Haiti ou de parents haitiens. Tous les responsables sont en fait nés en Haiti et un ménage seulement compte un membre non haitien.

- Voir la carte 1.

- En ce qui concerne l'enquete, la marge d'erreur d'échantillonnage autour d'une proportion de $50 \%$ pour l'ensemble de I'echantillon ( $N=152$ ) est de $\pm 8 \%$ Cette marge est de $\pm 20 \%$ lorsque 1 es zones sont comparées entre elles.

- Selon la definition de Statistique Canada, i.e. les fils et filles jamais mariés, quel que soit leur age, et vivant dans le même logement que leurs parents. 
structure familiale d'origine en Haiti, au processus d'immigration lui-mene, marqué par la separation temporaire ou permanente des membres de la famille, ou encore a l'insertion differentielle des femmes et des hommes d'origine haitienne dans la société québécoise.

Observée suivant la zone d'enquete, la composition des ménages haitiens peut rendre également compte de leur comportement résidentiel. La différence la plus importante entre les zones concerne d'une part, la distribution des familles sans enfants ou avec un seul enfant et d'autre part, celle des familles comptant deux enfants ou plus (Tableau 2). Ainsi la majorite des familles haitiennes résidant a st-Leonard $(56 \%)$ appartiennent au premier groupe, tandis qu'a Montreal et a Montréal-Nord, la majorité dentre elles $(70 \%)$ se retrouvent dans le second. On observe le meme rapport entre les zones pour l'ensemble de la population, avec toutefois des différences beaucoup plus faibles: $64 \%$ des familles de $5 t$-Léonard sont sans enfants ou en ont un seul comparativement a $56 \%$ des familles de Montréal et a $58 \%$ de celles de MontrealNord. Ces differences suggerent qu'il existe, pour les menages haitiens de chaque zone, des tendances générales qui caractérisent leur structure familiale et qui peuvent etre associees à celles qui distinguent également leurs conditions d'habitation.

La répartition des divers genres de ménage varie donc selon la zone observée, tant pour les menages haitiens considerés dans l'enquete que pour l'ensemble des ménages. Les besoins en logement different également selon le genre de ménage, que ce soit en termes despace, de configuration des pieces ou dorganisation de la vie domestique. Ainsi, malgréles différences entre zones, les exigences que pose la composition des ménages face a l habitat apparaissent au total plus lourdes pour les menages haitiens que pour l'ensemble des ménages, en ce qui concerne surtout la présence d'enfants jeunes, le partage d'un logement entre deux familles ou l'hébergement de personnes apparentés (grands-parents, cousins-cousines, neveux-nieces).

\section{Taille des menages et types de logement}

Comparée à la dimension des logements, la taille des ménages vient confirmer ce que leur composition a déja laissé entrevoir, à savoir la forte pression exercée en matière d'habitation sur une bonne partie des ménages haitiens dans les zones observés. La taille moyenne des ménages de l'enquete est de 4,1 personnes; les ménages de quatre personnes représentent le quart de 1 ensemble et ceux de cinq personnes et plus, environ $40 \%$. La majorité des ménages ont donc besoin, comme espace vivable, de logements comportant au minimum quatre ou cinq pièces. En fait, les quatre pieces predominent, puisqu'ils constituent prés de la moitie des logements comparativement a $35 \%$ pour les cing pieces et plus (Tableau 3). La dimension moyenne des logements 
TABLEAU 3

Caractéristiques des logements occupés par les ménages haitiens dans les zones d'enquete*

Caractéristiques des logements
Zones d'enquête

Montréal-Nord St-Léonard Montréal Ensemble des zones $\%$

$\%$

\section{Type de batiment residentiel}

Unifamilialb, duplex,

6,5 triplex

Multiplex

$1 \mathrm{~mm}$. a appartements

( 6 a 11 logements)

Imm. ¿ appartements

(12 log. et plus)

Autres immeublesc

$\begin{array}{rrrr}4,8 & 15,7 & 7,7 & 9,2 \\ 74,2 & 7,8 & 33,3 & 41,5 \\ 11,3 & 47,1 & 18,0 & 25,0 \\ 3,2 & - & 12,8 & 4,6\end{array}$

Total

100,0

100,0

100,0

100,0

Etage habité

Sous-sol

Rez-de-chaussée

38,7

41,2

23,1

35,5

(1er étage)

Deuxiène étage

Troisieme étage

et plus

16,1

19,6

10,3

15,8

30,7

29,4

41,0

32,9

14,5

9,8

25,6

i 5,8

Total

100,0

100,0

100,0

100,0
1-2

3

4

5

6-7
1,6

6,5

61,2

24,2

6,5
3,9

23,5

45,1

27,5

-

100,0

100,0

39

5,1

3,3

14,5

47,4

28,9

5,9

51

28,2

12,8

100,0

152

Total

100,0

Nombre de ménages*

62

Source: Données d'enquete, été 1982.

- Voir la carte 1; ce tableau est tiré de Berneche et Martin (1984).

- Comprend 3 maisons unifamiliales $(1$ a Montréal-Nord et 2 a Montréal).

- Immeubles conjuguant des fonctions résidentielles et commerciales.

d Les demi-pieces ne sont pas comptées.

- La marge d'erreur d'échantillonnage autour d'une proportion de $50 \%$ pour l'ensemble de l'échantillon $(N=152)$ est de $\pm 8 \%$. Cette marge est de $\pm 20 \%$ lorsque les zones sont comparées entre elles. 
est de 4,2 pieces, ce qui donne un rapport nombre de personnes/nombre de pieces eqal a $0,9 \%$, rapport se situant pres des limites de la surhabitation 27.

Les logements comptant cinq pieces et plus occupes par les menages haitiens se trouvent en plus forte proportion dans la zone de Montrél (51\%), alors que les petits logements ( 3 pieces et moins) sont sous-representes a Montreal-Nord (8\%) et surrepresentes a st-Leonard $(27 \%)$. C est aussi dans les zones de Montréal et de Montréal-Nord que resident la presque totalité des menages de six a neuf personnes rejoints dans 1 enquete (26 sur 28). Ils forment environ le quart des ménages résidant dans chacune de ces zones, mais seulement $4 \%$ de ceux vivant a St-Lénard (Tableau 2). Par ailleurs, les menages ne comptant qu une ou deux personnes se retrouvent surtout dans cette derniere zone et dans celle de Montréal oú ils composent respectivement $30 \%$ et $26 \%$ de l ensemble des ménages, contre $10 \%$ pour ceux habitant a Montréal-Nord. Ces differences entre zones peuvent en partie s'expliquer par le prix des ioyers 28 et le stock de logements de taille voulue disponibles dans chaque zone.

A l'echelle de lensemble de la population, le rapport nombre de personnes/nombre de pieces est de 0,63 pour les trois zones denquete. Il varie peu d une zone a l autre, soit 0,62 pour $5 t-L e ́ n a r d ~ e t ~ 0, b 4$ pour Montréal et Montréal-Nord. On compte en moyenne trois personnes par menage et quatre pieces et demie par logement. Il existe cependant, comme pour les autres caracteristiques observés. des variations a l'interieur meme des zones, a Montréal-Nord surtout où la dimension moyenne des logements passe de 4,1 pieces a 5,3 pieces selon le secteur de recensement29. Les rapports calcules restent toujours inférieurs a ceux obtenus pour les ménages haitiens de 1 enquéte, differenciant ainsi, quoique de façon imparfaite, leur utilisation de l espace habite.

Comme environ $80 \%$ des ménages privés résidant dans les memes zones, les ménages haitiens touchés par l enquete sont en majorité locataires de leur logement. Ils le sont toutefois dans une proportion plus forte, puisque

27 Lorsque ce rapport depasse 1, le loqement est generalement consideré comme surhabite. Dans son etude sur la population dorigine caraibéenne a Londres, T.R. Lee adopte plutôt la valeur de 1,5 comme seuil critique (1977: 115-120). Il n'y a donc pas dappréciation uniforme de ce rapport.

28 Selon le recensement de 1981, le loyer brut mayen pour un logement de 4,5 pieces en moyenne est plus elevé dans la zone dienquete situé a Montréal-Nord que dans celle de Montréal et légerement plus encore a St-Leonard (données fournies par A. Bastien).

29 Les secteurs 010.04 et 610.05 ou residait en 1981 le plus grand nombre de personnes nées en Haiti $(640$ et boo) sont aussi ceux ou les logements sont en moyenne de plus petite dimension $(4,1$ et 4,2 pieces). 
seulement quatre ménages, 50 it $3 \%$ de l'ensemble, sont propriétaires de 1 a maison où ils habitent. A l'interieur des zones d'enquete, la population haitienne se retrouve majoritairement dans des immeubles a appartements 30 . $A$ Montréal-Nord, les trois-quarts des ménages haitiens de l enquete résident dans des immeubles de $b$ a 11 logements, alors qu'a st-Léonard ils se partagent a peu prés egalement entre les grands immeubles de 12 logements et plus et les batiments résidentiels de type "plex" (Tableau 3). Dans la zone de Nontréal, la distribution des ménages haitiens selon le type de construction est beaucoup plus variée, suivant en cela le cadre bati.

Les logements occupés par les ménages haitiens au moment de l'enquete se situent surtout au sous-sol et au second etage des batiments residentiels (Tableau. 3). Les locations de sous-sol avoisinent les 40\% a Montréal-Nord et à St-Lénard, mais ne dépassent pas les $25 \%$ a Montréal ou dominent plutót les logements situés au second etage $(41 \%)$. Cette situation comporte des problemes spécifiques en matiere d'habitation, comme l'ont souligné plusieurs des personnes interviewés: problemes d'humidité et de chauffage dans les sous-sols, problemes 1 iés aux enfants (bruit, absence d'espace de jeu) pour les seconds etages.

Associées aux remarques faites sur la composition et la taille des ménages haitiens, celles concernant les types de logement qu'ils habitent soulignent donc le caractere distinctif de leur profil residentiel, marqué en plusieurs points par leur situation particulière d'immigrants récents. Un bref aperçu de la situation des membres de ces ménages face a l'emploi entraîne le méme type d'observations.

\section{Nombre de membres actifs dans les familles ot types d'emploi}

Les donnés de Statistique Canada pour la population totale fournissent une information sur le nombre de membres actifs selon la structure de la famille dans les ménages prives. La comparaison, en ce qui concerne les familles haitiennes de l'enquete, ne peut toutefois etre qu'indicative, car l'information recueillie dans cette enquete ne permet pas dadopter pour la population active une definition aussi stricte que celle retenue par

La comparaison avec l'ensemble des ménages n'est pas présentée, car les catégories retenues au recensement de 1981 pour le type de construction ne concordent pas avec celles de l'enquete; par ailleurs, il n'y a pas de donnés disponibles quant a l'etage habité, ni en ce qui concerne la distribution des logements selon le nombre de pieces. 
Statistique Canada31. Seront donc considerés, d'une part, les membres actifs occupant un emploi au moment de l'enquete et d'autre part, tous ceux occupant ou ayant déjá occupé un emploi (Tableau 4).

La premiere serie de résultats montre l'importance de la proportion des familles haitiennes se trouvant sans revenu d'emploi au moment de lenquete, soit $28,2 \%$. Les familles monoparentales, qui composent pres du tiers de ces familles, contribuent pour une large part a cette situation, puisque la moitie d'entre elles ne comptent aucun membre actif occupé.

Les familles époux-épouse de l'enquete ont presque toutes un membre qui occupe ou qui a déja occupé un emploi et $86 \%$ d'entre elles en ont au moins deux. Le decalage est donc marqué entre ces proportions et celles concernant uniquement les membres actifs occupés au moment de l'enquete. Ainsi, le passage, pour les familles comptant deux membres actifs ou plus, diune proportion de $86 \%$ a celle de $37 \%$ souligne a la fois la forte participation de ces familles à l'activité économique et l'importance accrue des familles haitiennes où les revenus d'emploi, au moment de l'enquete, ne proviennent plus que d'un individu $(45,6 \%)$.

La distribution du nombre de membres actifs selon la structure familiale, pour l ensemble de la population, se situe a mi-chemin entre les deux séries observés pour la population haitienne, ce qui concorde avec les définitions adoptés. A l'échelle de l'ensemble des familles, les proportions se rapprochent cependant, ne montrant plus qu une représentation légerement supérieure des familles sans aucun membre actif parmi la population totale $(13,2 \%)$ par rapport aux familles haitiennes (6, $0 \%$ pour les membres occupant ou ayant déjaccupé un emploij et l'inverse pour les familles comptant deux membres actifs ou plus $(55,1 \%$ contre $61,8 \%)$. Compte tenu des 1 imites de 1 a comparaison, ces faibles differences traduisent surtout la ressemblance des situations, tout au moins pour les familles considérées dans leur ensemble.

Selon cette comparaison, la participation des familles haitiennes a 1 activité économique est légerement plus élevée ou l'est autant que celle de toutes les familles vivant dans les memes zones, en admettant que presque tous les chômeurs et chômeuses haitiens recherchaient encore un emploi au moment de

31 Statistique Canada considere comme actives sur le marché du travail les personnes de 15 ans et plus qui, au cours de la semaine de recensement, faisaient partie de la population active occupee ou de la population active en chôlage. Les personnes en chômage devaient avoir cherché un emploi dans les quatre semaines précédant celle du recensement et être prêtes à travailler, ou prévoir reprendre un emploi ou en occuper un nouveau dans les quatre semaines suivant le recensement (Dictionnaire du recensement de 1981: 9). Ces précisions, qui peuvent réduire le nombre de personnes en chômage, n'ont pas été demandés dans l'enquête. 
TABLEAU 4

Structure de la famille et nombre de abmes actifs parmi les fanilles haitiennes de l'enquete et parni l'ensemble des fanilles résidant dans les meaes zones

\begin{tabular}{|c|c|c|c|}
\hline $\begin{array}{l}\text { Caracteristiques } \\
\text { des } \\
\text { fanilles }\end{array}$ & $\begin{array}{l}\text { es occupant un } \\
\text { i au moment de } \\
\text { enquete }\end{array}$ & $\begin{array}{l}\text { de l'enquête } \\
\text { zones) } \\
\text { Meabres occupant ou } \\
\text { ayant déjà occupé } \\
\text { un eaploi }\end{array}$ & $\begin{array}{l}\text { Enseable des fanilles } \\
\text { de recensenent dans } \\
\text { les zones d'enquête }\end{array}$ \\
\hline $\begin{array}{l}\text { Nombre de mabres actifs" } \\
\text { selon la structure de la } \\
\text { famille: } \\
\text { fanilies epoux-épouse } \\
\text { aucun aembre actif } \\
\text { un seul meabre actif } \\
2 \text { menbres actifs ou plus }\end{array}$ & $\%$ & k & $\begin{array}{l}4 \\
3 \\
3\end{array}$ \\
\hline Total & $100,0(101)$ & $100,0(101)$ & $100,0(14955)$ \\
\hline $\begin{array}{l}\text { Fanilles onoparentales } \\
\text { aucun membe actif } \\
\text { un nembre actif ou plus }\end{array}$ & $\begin{array}{l}50,0 \\
50,0\end{array}$ & $\begin{array}{l}16,7 \\
83,3\end{array}$ & $\begin{array}{l}33,6 \\
66,4\end{array}$ \\
\hline Total & $100,0 \quad(48)$ & $100,0(48)$ & $100,0(3645)$ \\
\hline $\begin{array}{l}\text { Enseable des fanilles } \\
\text { aucun mentre actif } \\
\text { un seul nembre actif } \\
2 \text { membres actifs ou plus }\end{array}$ & $\begin{array}{l}28,2 \\
45,0 \\
26,8\end{array}$ & $\begin{array}{r}6,0 \\
32,2 \\
61,8\end{array}$ & $\begin{array}{l}13,2 \\
31,7 \\
55,1\end{array}$ \\
\hline Total & $100,0 \quad(149)$ & $100,0 \quad(149)$ & $100,0(18600)^{c}=$ \\
\hline $\begin{array}{l}\text { Structure de la fanille } \\
\text { fanilles époux-épouse" } \\
\text { fanilles monorentales" }\end{array}$ & \multicolumn{2}{|c|}{67,8} & $\begin{array}{l}80,4 \\
19,6\end{array}$ \\
\hline Total & \multicolumn{2}{|c|}{$100,0(149)$} & $100,0(18600)^{c}$ \\
\hline
\end{tabular}

Sources: Données d'enquete, été 1982. Statistique Canada, Recensement du Canada 1981, catalogue nos 95-918 et 95-959 (secteurs de recensement).

- Voir la note 31.

- Fanilles principales (celles du/de la responsable du aénage) et fanilles secondaires.

- L'information disponible sur le nombre de meabres actifs concerne uniquenent les secteurs de recensement qui, d St-Léonard, couvrent un territoire plus vaste que celui coaposé des secteurs de dénombrement. Le nombre total de fanilles est donc supérieur à celui indiqué au tableau 2. Les proportions varient cependant tres peu: ainsi, parni les 17425 fanilles du territoire habituelienent considéré (Tableau 2), on compte $80,1 \%$ de familles époux-épouse et $19,9 \%$ de fanilles monoparentales. 
lenquetes2... Avec un niveau semblable d activité économique, les familles haitiennes ont cependant une charge familiale plus forte que celle de lensemble des familles, puisquelles comptent une proportion plus élevé d'enfants agés de moins de 15 ans33. La comparaison montre également que les familles monoparentales, tant celles faisant partie de lenquete que celles de l'ensemble de la population, connaissent une situation nettement plus difficile en termes de revenu d'emploi que les familles époux-epouse.

Dans l'ensemble des zones d'enquete, le profil occupationnel des membres actifs au sein des familles haitiennes, soit 145 femmes et 128 hommes au total, est particulierement marqué par la predominance des personnes travaillant ou ayant travaille dans le secteur industriel, que ce soit dans la transformation (aliments et boissons, textile), l usinage (métaux, bois) ou la fabrication, le montage et la reparation de produits (articles en tissu, matieres plastiques, materiel électronique, etc.). On y retrouve $67,4 \%$ des femmes et $61,1 \%$ des hommes. Ce groupe de travailleuses et de travailleurs est nettement moins important parmi la population totale des zones denquete, car il $n$ inclut que $25,3 \%$ des femmes actives et $26,9 \%$ des hommes actif $5^{34}$.

Dans les zones d'enquete, l'ensemble de la population active féminine se caractérise par un trés fort pourcentage de femmes travaillant comme employés de bureau $(36,5 \%)$; il en va autrement pour les femmes haitiennes qui se partagent également entre les emplois de bureau $(9,2 \%)$, ceux en médecine et santé $(10,0 \%)$ et les services $(10,6 \%)$. L'éventail des professions est plus large pour la population active masculine qui se differencie moins nettement des travailleurs haitiens, sauf pour le secteur du batiment qui ne compte personne dans l'enquete, mais où se retrouve le dixième de tous les hommes actifs. Frès du dixieme d'entre eux travaille aussi dans les transports, soit une proportion semblable a celle des travailleurs haitiens $(10,3 \%)$ qui se concentrent cependant tous dans un seul domaine, celui du taxi.

La population haitienne considérée dans l'enquete est donc beaucoup plus ouvriere que ne l'est l'ensemble de la population résidant dans les memes zones. Par ailleurs, tout comme la population active de ces zones, elle se retrouve peu dans les postes de direction, dans les professions techniques et sociales ou dans l'enseignement. Ces trois grands groupes rassemblent en effet seulenent $4 \%$ des travailleuses et travailleurs haitiens et $13 \%$ de la population active totale.

32 En fait, $6 \%$ des personnes ayant déja occupé un emploi suivaient des cours ou etaient considerées comme étudiantes au moment de l'enquete. Si l'on soustrait ces personnes du nombre des membres actifs occupant ou ayant déja occupé un emploi, les pourcentages ne sont que légerement modifiés, se rapprochant de ceux obtenus pour l'ensemble de la population.

33 Parmi les familles haitiennes, $79,3 \%$ des enfants sont agés de moins de 15 ans comparativement a $55,3 \%$ parmi l'ensemble des familles.

$34 \mathrm{Si}$ on ajoute le groupe des "autres travailleurs", ces proportions s'élévent a $30,3 \%$ pour les femmes et a $33,9 \%$ pour les hommes. 
Les resuitats observes montrent donc que la participation des familles haitiennes a lactivite économique est importante, mais que leur situation effective en termes de revenus demploi au moment de l enquete est difficile. Cette situation peut etre liee au profil occupationnel de leurs membres, marque par la predominance des travailleurs et travailleuses en usine. dans des secteurs d'activite fortement touches par la recession economique 35 . Il 5 'agit d'une situation densemble, présentant selon les zones d'enquete certaines variations, dont l'interprétation demande cependant une analyse plus fine des resultats.

\section{CONCLUSION}

En réponse aux questions posés au debut de cet article, nous rappellerons brievement les principales tendances qui se degagent des themes etudies. Nous en tirerons les elements permettant d'associer aux regroupements de population haitienne, comme occupation specifique de lespace, la position particuliere dune fraction importante de cette population comme clientele sur le marche du logement et comme main-d oeuvre sur le marché du travail.

Les zones observees, ou se regroupe une large part de la population immigrante haitienne, ne forment pas un bloc homogene et distinct dans l espace montréalais. Chacune combine de manière specifique des élements qui en caracterisent le cadre bati et lorganisation spatiale. De façon commune toutefois, ces zones $\Xi$ inscrivent par leurs caracteristiques residentielles dans un espace urbain associé a la péripherie immédiate du centre*ville ou aux proches banlieues. Ce sont aussi des zones ou la population immigrante est relativement importante, notamment a Montreal et ¿ st-Leonard. Elie l est un peu moins dans la zone de Montréal-Nord, mais se compose alors d une plus forte proportion d'immigrantes et d'immigrants haitiens.

La population immigrante haitienne qui reside dans les zones dienquete appartient en grande majorité a la "seconde vague" de l'immigration haitienne. Les trois quarts des personnes interviewés sont en effet arrivees au cours des années 1971 a 1978 . Frès de la moitié des repondants et repondantes sont entrés au Canada comme touristes (visiteurs) et se sont ainsi trouvés concernes par les operations gouvernementales visant a regulariser les differents statuts d'entrée conformément a la nouvelle loi d'immigration adoptee en 1978.

La situation des ménages haitiens au moment de l enquéte, en termes de logement et demploi, exprime a la fois le caractere relativement recent de leur immigration et leur insertion particuliere dans l'espace socio-économique

35 Le secteur des services et celui de la "fabrication, montage et réparation de produits" (textile, fourrure et cuir, entre autres) se caracterisent par de faibles niveaux de remuneration et un nombre eleve d heures de travail hebdomadaires; ce sont aussi des domaines "ou la situation concurrentielle précaire defavorise les employes en terme de stabilité et sécurité d'emploi" (Normandeau et Fiché, 1983: 85). 
montréalas. La taille et la composition des ménages haitiens, comparees a celles de l'ensemble des ménages, se caractérisent en effet par un nombre pius elevé de membres et par une importance accrue des families comptant des enfants à 1 a mason. La dimension des logenents occupes par ces ménages est cependant proche de ceile de l'ensemble des logements prives, ce qui donne un rapport rombre de personnes/nombre de piéces plus élevé pour les ménages haitiens. Cette densite plus forte doccupation peut etre lié a la reconstitution des familles ou a l'hebergement dautres personnes arrivant a llontréal. La composition différente des ménages haitiens doit aussi etre situee dans sa dimension socia-culturelle, soit la structure méme de la famille haitienne et sa transformation en situation dimmigration.

Heme sils comptent tous deux une faible proportion de postes administratifs et professionnels, les profils des emplois pour la population hätienne et pour l ensemble de la population traduisent une situation fort differente. En effet, la majorite des travailleurs et travailleuses parmi les menages haitiens sont ouvriers et ouvrieres en lusine, alors que ces emplois regroupent environ le tiers de la population active residant dans les zones denquete. Il y a donc, pour la population haitienne de ces zones, une nette concentration sur le marché du travail, dans des categories demploi qui, surtout chez les femmes, sont souvent considerées comme vulnerables lle texti le par exemplei.

Leurs besoins en matiere d'habitation, combines d leurs ressources economiques restreintes, placent donc la plupart des menages haitiens de 1 enquete parmi les groupes sociaux les plus touches par la crise du logement. Dans ieur recherche d un iogement, les immigrantes et les immigrants haitiens doivent aussi faire face aux problemes que pose la discrimination ethnique ou raciale. Devant ces contraintes. ie recours aux réseaux de parents et/ou d'amis. d'aide et/ou d'information36, peut constituer un moyen de minimiser les risques et les difficultes, tout en amenant les individus vers des secteurs déje connus de la ville, favorisant alors les regroupements de population haitienne. Le comportement residentiel des menages haitiens vivant dans les zones dienquéte est ainsi marqué, comme le montrent ces tendances, par leur double situation comme groupe socio-economique désavantage et comme groupe Ethnique minoritaire.

36 La dimension transnationale de ces réseaux (Etats-unis, Haiti, Quebec) et leur imoortance pour la population haitienne résidant a fiontréal ont ete mises en evidence dans le cadre dun autre projet du centre de recherches caraibes, "Familie, travail et reseaux migratoires" (sous la direction de 5. Larose, H. Labelle et V. Fichei. 


\section{RÉFÉRENCES BIBLIOGRAPHIQUES}

ALALUF, H., 1982. La culture dorigine et la culture des migrants. Rapport géneral. Frojet no 7 du CDCE "L'education et le développement culturel des migrants". Strasbourg, Conseil de la cooperation culturelle.

BAROU, J., 1980. "Immigration et enjeux urbains". Pluriel, Débat, $24,3-20$.

BEFNECHE, F., 1983. "Les caractéristiques socio-démographiques de l'immigration caraibeenne au Quebec au recensement de $1971^{\prime \prime}$, in Victor Piché et al., L'inigration caräibenne au canada et au duebec: aspects statistiques. Montréal, Centre de recherches caraibes, Université de Montréal, 93-112.

BERNECHE, F. et MARTIN, J.C., 1984. "Immigration, emploi et logement: la situation de la population haitienne dans certaines zones de la région métropolitaine de Montréal". Anthropologie et sociétés, 8, 2, 5-29.

BEFNIER, B., 1978. "Les phenomenes urbains dans le capitalisme actuel". Cahiers de geographie du Quebec, 22, 56, 189-216.

BoRgogno. C., 1978. "L'espace de l'immigration". Pluriel, Débat, 14, $43-52$.

CASTELLS, M., 1977. La question urbaine. Paris, Maspero, edition de 1972 revue.

CHOKO, M.H., 1980. Crises du logenent a Montreal (1860-1939). Montreal, Editions coopératives Albert Saint-Martin.

CHOKO, M.H., 1981. "Pour une 'statistique du vécu' des travailleurs en matiere de logement". Interventions critiques en econolie politique, Dossier: Environnement, printemps/ete, 111-124.

DEJEAN, F., 1978. Les Hä̈tiens au Quebec. Montréal, Les Fresses de 1 Université du Québec a Montréal.

HURBON, L., 1982. "La fuite du peuple haitien: ou les causes de la migration actuelle". Collectif Paroles, février/mars, 16, 34-37.

KEMFENEERS, M. et PICHE, V., 1983. "Caractéristiques socio-démographiques de l'immigration caraibéenne au Québec: 1968-1980", in Victor Fiché et al., Limigration caraibentie au Canada et au Quebec: aspects statistiques. Montréal, Centre de recherches caraibes, Université de Montréal, 61-71.

LAVIGNE, G., 1981. "Le pouvoir ethnique: ses assises et ses objets", in ACSALF: Colloque 1979, La transforation du pouvoir au Québec. Montréal, Editions cooperatives Albert Saint-Martin, 171-182.

LEE, T.R., 1977. Race and Residence. The Concentration and Dispersion of I igrants in London. Oxford, Clarendon Press. 
MARIE, M. et DOS SANTOS,J.R., 1973. "L'immigration et la ville". Espaces et Societes, février, $8,23-36$.

MATHEWS, 6., 1978. L'impact de l'imigration sur le arché du logement dans la région etropolitaine de Hontreal de 1961 a 1981 . Gouvernement du Quebec, ministere de l'Immigration. (Etudes et documents 6)

NORMANDEAU, L. et PICHE, V., 1983. "L'emploi projeté des travailleurs et travailleuses caraibéns-nes au Québec", in Victor Piché et al., Limagration caraibenne au Canada et au Quebec: aspects statistiques. Montréal, Centre de recherches caraibes, Université de Montréal, $73-91$.

POCHE, B., 1975. "Mode de production et structures urbaines". Espaces et Sociétes, novembre, $16,15-30$.

FOLESE M., HAMEL C. et BAILLY A., 1978. La géographie résidentielle des inigrants et des groupes ethniques: Hontréal, ig7i. Montréal, I.N.R.S. Urbanisation. (Etudes et documents 12 ).

PORTES, A. et WALTON,J., 1981. Labor, CIass and the International systen. Studies in Social Discontinuity. New York, Academic Press. 


\section{RÉGUHÉ - SUMMARY - RESUMEN}

\section{BERNĖCHE Francine - Inagration et espace urbain. Les regroupenents de population haitionne dans Is region attropolitaine de Montreal}

La population haitienne conpte parai les groupes innigrés les plus récents de la région aétropolitaine de Montréal. Dix fois plus nonbreuse en 1981 qu'en 1971, cette population d'environ 30000 personnes réside en ajorité (80\%) dans trois des quinze municipalités de la région montréalaise: Montréal-Nord, St-Léonard et Montréal. Afin d'identifier les causes et les aécanisnes des regroupenents de population haitienne a l'intérieur de ces municipalités, une enquete a été menée au cours de l'eté 1982 auprès de 152 ménages haitiens résidant dans des zones de concentration. Les besoins de ces aénages en atiere de logement different de ceux de l'ensenble des aénages vivant dans les aenes zones: les aénages haitiens se caractérisent par un noabre plus elevé de aeabres et par l'iaportance accrue des fanilles comptant des enfants à la maison, enfants qui sont aussi plus jeunes. Les logenents qu'ils occupent se situent pour la plupart dans des imeubles a apparteaents, le plus souvent au sous-5ol ou au second étage des batiments résidentiels; ce sont surtout des logements de taille aoyenne (quatre ou cinq pieces), ce qui donne une densité d'occupation plus forte pour les ménages haitiens que pour l'ensenble des énages. La situation socio-écononique de ces ménages leur iapose égalenent des contraintes particulières, puisque leurs aembres actifs sont davantage concentrés dans un aene secteur du aarché du travail que ne l'est la population active des aémes zones: les deux tiers sont ouvriers et ouvrières en usine. Ces caractéristiques situent donc la population haitienne des zones d'enquete parai les groupes sociaux désavantagés sur le marché du logement et les plus susceptibles d'etre touchés par la crise actuelle.

BERNĖCHE Francine - Imigration and Urban Space. The Foration of Groups of Haitians in the Metropolitan Region of Montreal

The Haitian population is among the most recent groups of imigrants of the Metropolitan Region of Montreal. Ten times as numerous in 1981 than in 1971, this population of about 30000 people lives wainly $(80 \%)$ in three of the fifteen aunicipalities of the Montreal region: North-Montreal, St-Leonard and Montreal. In order to identify the causes and the mechanisms of the foration of groups of Haitians within these municipalities, a survey of 152 Haitian households living within the areas of concentration was conducted during the summer of 1982 . The Haitian households have different housing needs than the whole of the households living in the same areas: Haitian households are characterized by a larger number of meabers, and by the increased importance of families with children living at hone, children who are also younger. The housing units that they occupy are mostly found in apartment buildings, most often in basements or on the second floor of residential buildings; these are nostly mediun size flats (four or five rooms) which means a higher occupation density for Haitian households than for the whole of the households. The socio-econonic situation of these households also iaposes other particular constraints, since their work force is more concentrated in one sector of the labour warket than the work force of the same areas: two-thirds are factory workers. Therefore, these characteristics place the Haitian population of the areas surveyed, anong the social groups that are at a disadvantage in the housing market and which are oost liable to be affected by the present (econonic) crisis. 


\section{BERNĖCHE Francine - Inaigración y espacio urbano. Los agrupanientos de población haitiana on el írea aetropolitana de Montreal}

La población haitiana se coloca entre los grupos de inaigrantes aás recientes del área metropolitana de Hontreal. Esta población cuenta con alrededor de 30000 personas, o sea diez veces ás numerosa en 1981 que diez años antes, cuya ayoría $(80 \%)$ reside en tres de los quince aunicipios de la zona regiomontana: Montreal-Norte, San Leonardo y Montreal. Con objeto de identificar las causas y los mecanismos del agrupamiento de población haitiana en el seno de estos sunicipios, se llevó a cabo una encuesta durante el verano de 1982, en 152 hogares haitianos ubicados en las zonas de ayor concentración. Las necesidades de estos hogares en cuestión de vivienda difieren de las del conjunto de hogares que se encuentran en las mismas zonas: los hogares haitianos se distinguen por un ayor número de mieabros, así cono por el número relevante de fanilias que cuentan con niños dentro de la casa, los cuales son incluso pés pequeños. La ayor parte de las viviendas que ocupan los haitianos se ubican en edificios de departamentos, situandose a menudo en el sótano o en el priaer piso de estos conjuntos residenciales; generalaente son viviendas de taaño mediano (de cuatro o de cinco piezas) lo que produce un indice de hacinauiento mucho ayor entre los hogares haitianos que en el resto de los hogares. Asinisao la situación socioéconomica de estos hogares los somete a presiones particulares, ya que sus miembros activos están principalmente incorporados en el uercado de trabajo, a diferencia de la población activa de las misas zonas: dos terceras partes son obreros u obreras de fábricas. Estas peculiaridades colocan así a la población haitiana de las zonas estudiadas, dentro de los grupos sociales aenos privilegiados dentro del mercado de la vivienda, adenás de ser los ás susceptibles en cuanto a ser afectados por la crisis actual. 